\title{
Effects of Variation of the Organic Solar Cell Layers' Dimensions on Electrical Charge Carrier Transfer
}

\author{
Nasrin Sepahvand and Mohsen Bahrami* \\ Department of Physics, University of Lorestan, Khorramabad, Iran \\ *Corresponding Author's Email: Bahrami.m@lu.ac.ir \\ Regular paper: Received: Nov. 7, 2020, Revised: Mar. 7, 2021, Accepted: May. 15, 2021, \\ Available Online: May. 17, 2021, DOI: 10.52547/ijop.15.1.3
}

\begin{abstract}
In this work, the effect of changing the dimensions of the layer structure on the collection of electrical charge carriers which been produced in the thin film composed of P3HT $^{1}$ and PCBM $^{2}$ that is between two electrodes, using the Monte Carlo numerical simulation with Bortez, Callus and Lebowitz algorithms, with checkered structure and different dimensions $60 \times 15 \times 5$ sites, $60 \times 30 \times 5$ sites, have been the conditions of the layers. At first, the average number of electrons and holes produced on the cathode and anode electrodes in two stages (simultaneous injection of excitons, without and with the presence of deep traps) was calculated and it was concluded that, by increasing layer width, the average number of electrical charge carriers collected on the electrodes has decreased, which has a direct impact on production of layer circuits and solar cell performance. Finally, the amount of external quantum efficiency of the layers was also calculated. In $60 \times 15 \times 5$ sites layer, in two stages without and with the presence of traps - the average value of external quantum efficiency $52.3 \%$ and $42.43 \%$ was obtained and in $60 \times 30 \times 5$ sites layer, the value of $42.43 \%$ and $37.9 \%$ was calculated.
\end{abstract}

KEYWORDS: External quantum efficiency, Monte Carlo, Organic semiconductor thin film, Solar cell.

\section{INTRODUCTION}

According to human needs and the use of clean and renewable Energies and the limitation of fossil fuels and environmental pollution caused by them, since decades ego, solar energy and

${ }^{1}$ Poly(3-hexylthiophene) converting into electrical energy by solar cells, has been considered by researchers [1]-[3]. Organic semiconductors materials are widely used in solar cells due to the deposition of nanometer thin films, high absorption coefficient and conductivity of electrical charge carriers [4]-[7]. Organic solar cells because of low cost, high flexibility, fast and easy largescale fabrication method large-scale solar cell can be a suitable alternative to new generations [8]-[10]. Computational modeling and simulation are effective ways to optimize and deliver the best solar cell performance without having to produce them even for the first time. These simulations give us insight into how the solar cell is structured, which can influence the generation, transmission, and collection of electrically charged carriers on the electrodes that help generate external circuit current and the efficiency of solar cells [11]-[13].

So in this article using the jump rate electrical carriers charge by Miller-Abraham and simulation through Monte Carlo computational algorithm, our aim is to investigate the effect of various factors on the transfer of electric charge carriers and to find a relationship between the internal structure of a solar cell and the external quantum efficiency of a thin layer in an organic solar cell.

\section{MODEL AND CALCULATION}

At first we consider a grid with a checkered structure for the active layer as shown in Fig. 1. To simulate the electrical charge transfer on a

\footnotetext{
${ }^{2}$ Phenyl-C61-butyric acid methyl ester
} 
three-dimensional grid, we follow the steps presented in flowchart of Fig. 2.

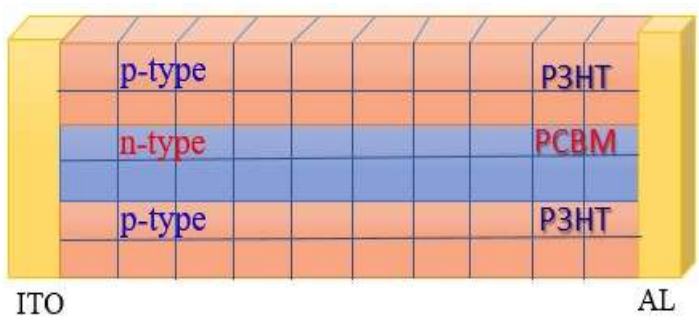

Fig. 1. The checkered structure of organic solar cell

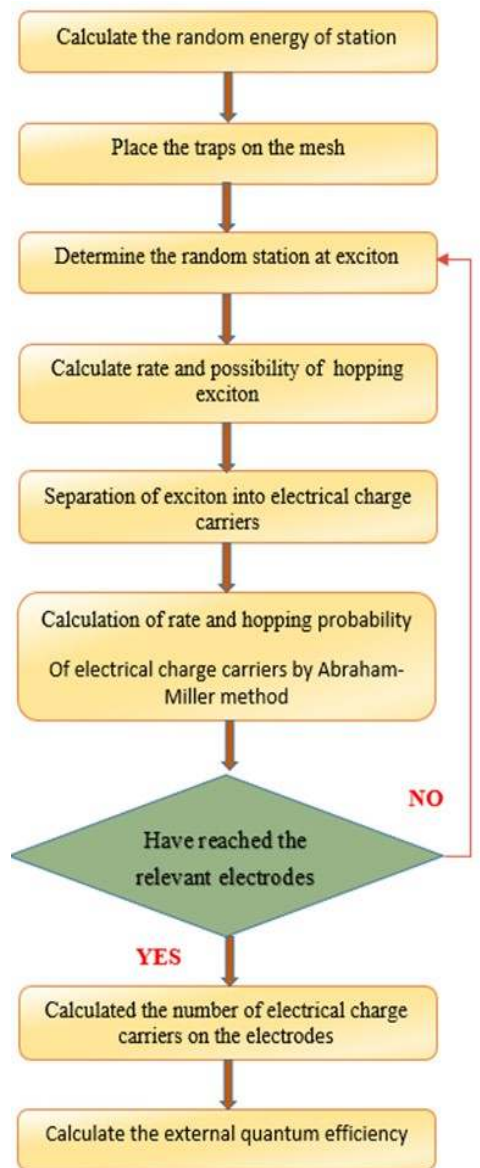

Fig. 2. Flowchart of Monte Carlo simulation operations.

\section{Step 1:}

First, we consider an exciton in a random location as a sign of photon radiation on the active layer [14].

Step 2:
The energy level of each position, which represents the $\mathrm{HOMO}^{1}$ and $\mathrm{LUMO}^{2}$ levels of that position, is generated from the Gaussian function of Eq. (1), which is randomly generated by the center of the LUMO level in the electron transfer medium and the HOMO level in the Gaussian $(\sigma)$ hole transporter can be calculated [15], [16].

$$
g(E)=\frac{1}{\sqrt{2 \pi \sigma^{2}}} \exp \left(-\frac{\left(E-E_{0}\right)^{2}}{2 \sigma^{2}}\right)
$$

Step 3:

The hopping rate $\left(W_{i j}\right)$ between different points of the lattice is calculated through the following equation:

$$
\begin{aligned}
& w_{i j}=w_{e}\left(\frac{R_{0}}{R_{i j}}\right)^{6} F\left(\Delta E_{i j}\right), \\
& F\left(\Delta E_{i j}\right)= \begin{cases}\exp \left(-\frac{\Delta E_{i j}}{K_{B} T}\right) & \left.\Delta E_{i j}\right\rangle 0 \\
1 & \Delta E_{i j}\langle 0\end{cases} \\
& W_{e(n)}=\frac{6 k_{B} T \mu_{e, h}}{q a^{2}} \exp (2 \gamma a), \\
& \Delta E_{i j}=E_{j}-E_{i},
\end{aligned}
$$

where $E_{i}$ and $E_{j}$ are the energy of the charge carriers of the two sites $i$ and $j ; k_{B}, T, \gamma, W_{e}$, and $R_{0}$ are respectively the Boltzman constant, temperature, amount of replacement, hopping frequency, and replacement radius of electrical charge carriers. $R_{i j}$ is the distance between the hopping position and $\mu_{e, h}$ is the mobility of the electrical charge carriers. The lattice constant $a$ is equal to $1 \mathrm{~nm}$ [15], [17].

\section{Step 4:}

Separation of excitons at the common boundary between electrical charge carriers and their

${ }^{2}$ Lowest unoccupied molecular orbital 
hopping rate is done by the Miller-Abraham method [18]:

$$
W_{i j}=\left\{\begin{array}{ll}
W_{e}\left(-2 \gamma R_{i j}\right) F\left(\Delta E_{i j}\right), & \left.\Delta E_{i j}\right\rangle \\
W_{e}\left(-2 \gamma R_{i j}\right), & \Delta E_{i j} \leq 0
\end{array},\right.
$$

Step 5:

Calculation of the hopping probability of electrical charge carriers:

$P_{K}=\frac{W_{i j}}{W_{\text {total }}}$,

where $W_{\text {total }}$ is the collection of hopping rate to nearby locations. A value between zero and one is obtained for the probability of hopping.

Step 6:

Finally, the average electric charge carriers located on the parallel layers of the electrodes and the external quantum efficiency (EQE), that is to say, the ratio of the number of electrons or holes collected in the corresponding electrode to the number of excitons injected, are calculated [19], [14].

\section{RESUlTS AND DISCUSSION}

In simulation, we consider the solar cell as an array of cubes (square), which means, the active layer splits into very small cubes (square) where the cubic lattice has $n_{x} \times n_{y} \times n_{z}$ position in $\mathrm{x}, \mathrm{y}$ and $\mathrm{z}$ directions. In the present work, we have considered $\mathrm{PCBM}^{1}[6]$ one of the fullerin derivatives, with the mobility of $3 \times 10^{-3} \mathrm{~cm}^{2} v^{-1} \mathrm{~s}^{-1}$ as a n-type semiconductor and $\mathrm{P}_{3} \mathrm{HT}^{2}$ as a p-type semiconductor, with a high mobility of about $2 \times 10^{-4} \mathrm{~cm}^{2} \mathrm{v}^{-1} \mathrm{~s}^{-1}$ (Fig. 1). These materials are used because they are suitable electron and hole transmitters, the properties of them are given in Table 1 [14], [22], [20].

The transparent and electrically conductive electrodes of the anode (indium-tin oxide ${ }^{3}$ ) and

${ }^{1}$ Phenyl-C61-butyric acid methyl ester

${ }^{2}$ Poly(3-hexylthiophene) the cathode (aluminum ${ }^{4}$ ) are placed around the layer to collect the electric charge carriers, which their energy levels with the active layer are plotted in Fig. 4 [24].

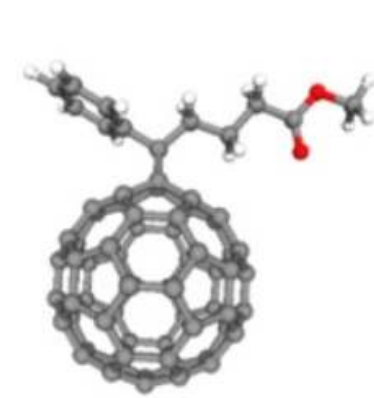

(a)

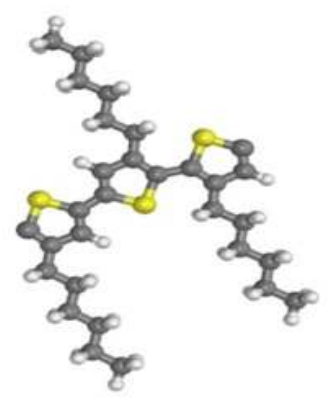

(b)
Fig. 3. (a) PCBM; (b) P3HT

Table 1. Parameters for the P3HT and PCBM [14].

\begin{tabular}{|c|c|c|}
\hline Property & P3HT & PCBM \\
\hline $\begin{array}{c}\text { Charge carrier mobility, } \mu \\
\left(\mathrm{cm}^{2} \mathrm{v}^{-1} \mathrm{~S}^{-1}\right)\end{array}$ & $2 \times 10^{-4}$ & $3 \times 10^{-3}$ \\
\hline $\begin{array}{c}\text { Energy width of density of } \\
\text { states, } \sigma_{E}(\mathrm{eV})\end{array}$ & 0.063 & 0.063 \\
\hline $\begin{array}{c}\text { Transmission energy of } \\
\text { carriers }(\mathrm{eV})\end{array}$ & $\begin{array}{l}E_{\mathrm{HOMO}}=5.2 \\
E_{\mathrm{LUMO}}=3\end{array}$ & $\begin{array}{l}E_{\mathrm{HOMO}}=6.1 \\
E_{\mathrm{LUMO}}=3.7\end{array}$ \\
\hline
\end{tabular}

To perform the simulation, an environment with dimensions of $60 \times 15 \times 5$ sites, with a checkered structure (consisting of three layers), the length of each layer is $60 \mathrm{~nm}$ and its width is $15 \mathrm{~nm}$ and its thickness is $5 \mathrm{~nm}$, and another environment is similar to that but with dimensions of $60 \times 30 \times 5$ sites, whose width we have doubled, is taken into account. We assume that the molecules P3HT and PCBM are located at points (nodes) of this lattice with a constant of $1 \mathrm{~nm}$. In $\gamma$ numerical calculations, the displacement rate is often equal to $2 \mathrm{~nm}^{-1}, K_{B} T$ is equal to. $25 \mathrm{eV}, \quad R_{0}$ is the exciton displacement radius, and $R_{i j}$ the distance between the sites $i$ and $j$ is also considered to be $1 \mathrm{~nm}$. Numerical calculations are performed in two steps as follows. At each stage, a diagram of the electrical charge carriers collected at the respective electrodes is drawn according to the number of charge carriers produced at common boundary between the two donor-acceptor

${ }^{3}$ Indium tin oxide: ITO

${ }^{4}$ Aluminum: Al 
materials, also a diagram of the external quantum efficiency according to descending excitons is drawn in both structures and compare with each other.

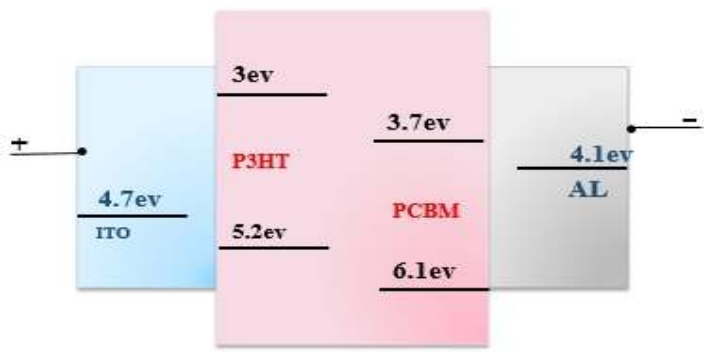

Fig. 4. The place of thin film, the electrodes and their energy levels.

\section{A. Simultaneous injection of excitons on the layer}

We considered the injection of a limited number of excitons $(10,30,50,80,100,116)$ in the first step of the simulation process, given that each site (molecule) can only receive one exciton or electrical charge carrier.

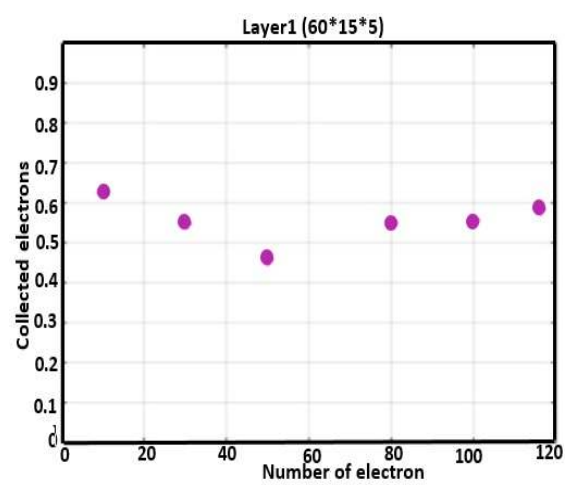

(a)

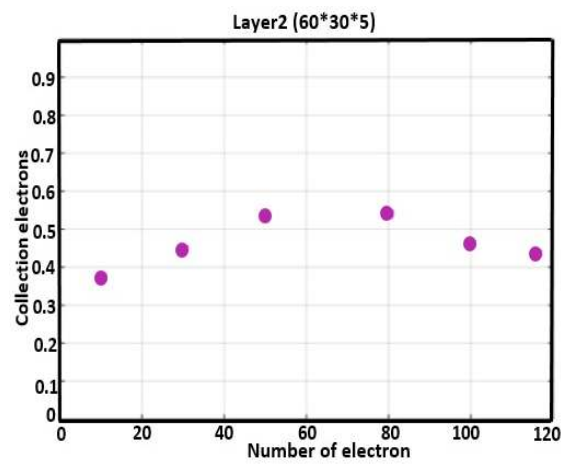

(b)

Fig. 5. Collected electrons at the cathode according to the number of produced electrons at the interface between the two layers. (a) $60 \times 15 \times 5$ site. (b) $60 \times 30 \times 5$ site.
Due to the difference in energy of sites of the lattice and different mobility of charge carriers in the active layer, the probability of hopping from one position to another is unequal, so we expect that the number of charge carriers collected at the electrodes is different in term of numerical results. Finally, by limiting the acceptance of sites in receiving the electrical charge carriers and the only loss factor (reaching the unrelated electrode charge carriers), the number of collected electrons on the cathode electrodes and the number of collected holes on the anode electrodes in the layer of $60 \times 15 \times 5$ sites is more obtained from the $60 \times 30 \times 5$ sites layer. This difference can be seen in Figs. 5 and 6.

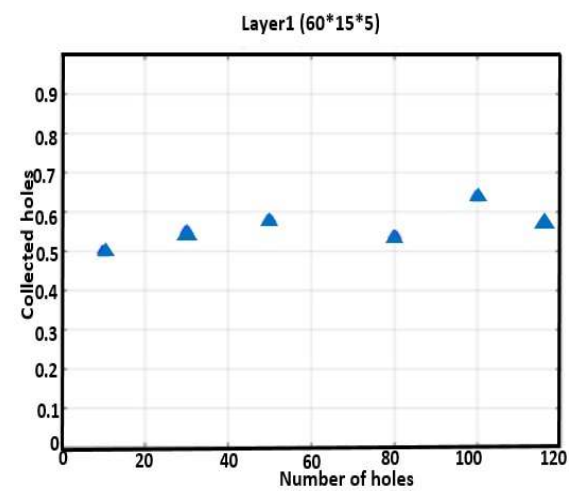

(a)

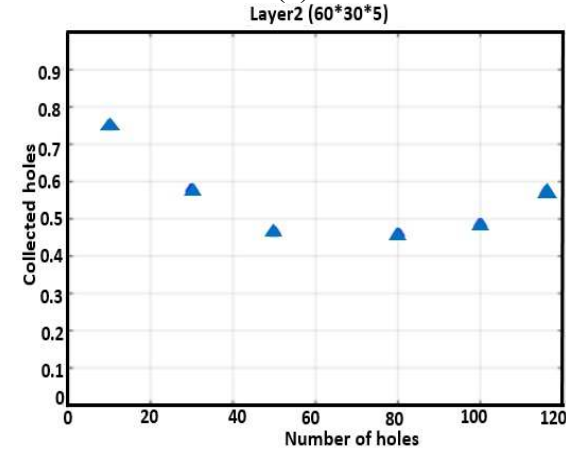

(b)

Fig. 6. Collected holes at the anode according to the number of produced holes at the interface between the two layers. (a) $60 \times 15 \times 5$ site. (b) $60 \times 30 \times 5$ site.

According to Eq. (4), the mobility followed by the rate and probability of hopping for electrons is less than holes. It seems to be the reason for difference in the behavior of electrons and holes. In addition to the results obtained, if there is more free space for the particles and the hopping rate is also high, the desired particles are more likely to reach the relevant electrode. 
Under these conditions, the average external quantum efficiency of layer $60 \times 15 \times 5$ sites was $52.3 \%$ and layer $60 \times 30 \times 5$ sites was $42.43 \%$.

\section{B. Applying deep traps \& trapping for electric charge carriers}

In the second stage of the simulation, the location of deep traps and the energy of the places where the traps are located were randomly considered with the energy center of the deep traps and the Gaussian width $\sigma=0.06$.

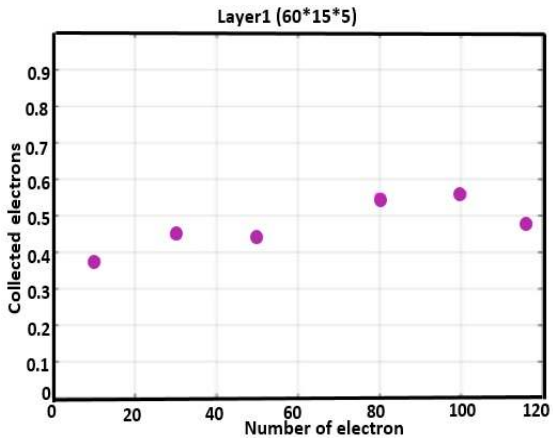

(a)

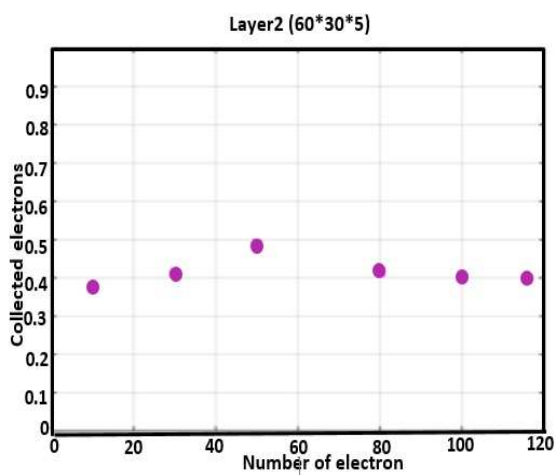

(b)

Fig. 7. Collected electrons at the cathode according to the number of produced electrons at the interface between the two layers. (a) $60 \times 15 \times 5$ site. (b) $60 \times 30 \times 5$ site.

The energy level of the traps is equal to the average energy level of the donor or acceptor of the electrical charge of the active layer of the solar cell. Some electrical charge carriers may be trapped by traps during a hopping or transfer movement or may remain their permanently, which ultimately cannot help transmit circuit current, which is why it reduces the collected electrons and holes in Figs. 3 and 4 compared to Figs. 5 and 6. In addition, from the data in Table 1, it can be seen that the energy distribution center of the traps is farther from the energy distribution center of the HOMO and
LUMO levels for the movement of the holes than the electrons, which causes the holes trapped in traps will much less able to retransmit current, which could be a reason for a greater drop in collected holes than electrons. In this work, the number of traps is 0.1 , the total number of points in each row in the layer.

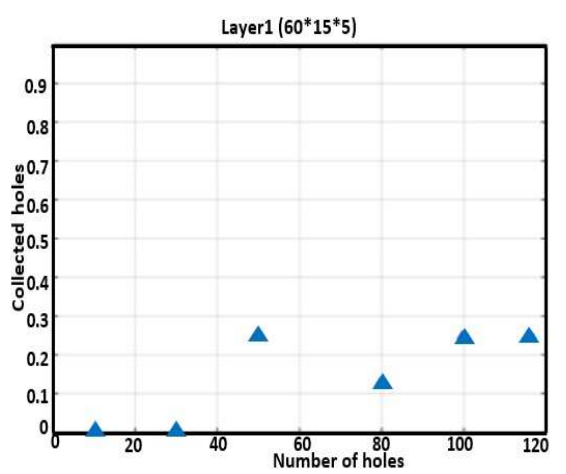

(a)

Layer2 $(60 * 30 * 5)$

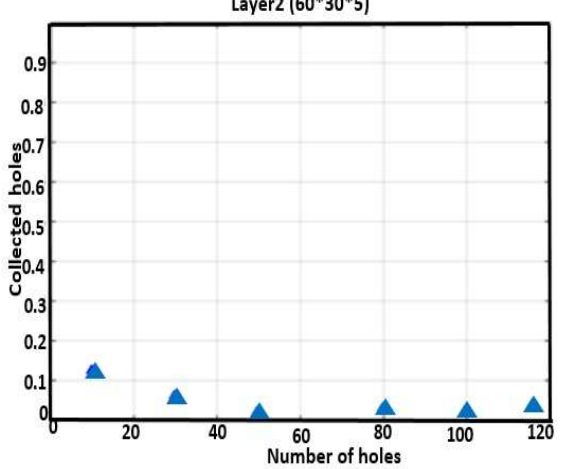

(b)

Fig. 8. Collected holes at the anode according to the number of produced holes at the interface between the two layers. (a) $60 \times 15 \times 5$ site. (b) $60 \times 30 \times 5$ site.

Table 2. Comparison of the average number of electrical charge carriers of different structures

\begin{tabular}{|l|c|c|}
\hline \hline Stage & Electron & Hole \\
\hline \hline The first layer & 36 & 32 \\
\hline The second layer & 31 & 28 \\
\hline
\end{tabular}

\begin{tabular}{|l|c|c|}
\hline \hline Stage & Electron & Hole \\
\hline \hline The first layer & 30 & 13 \\
\hline The second layer & 23 & 9 \\
\hline
\end{tabular}

This dimension variation shows a direct relationship between the better performance of the first structure and the number of electric charge carriers that play an important role in generating circuit current. A comparison of the above mentioned results is shown in Table 2 . 
The efficiency of solar cells, which is the result of the separation, transfer and collection of electric charge carriers, is directly related to their average number. As can be seen in Fig. 9, the efficiency of the solar cell decreases as the charge carriers become trapped in the traps and also as the distance from charge carriers to the related electrodes increases due to the increase in layer size, and layer $60 \times 15 \times 5$ sites, with a value of $42.63 \%$, has a higher external quantum efficiency than the layer of $60 \times 30 \times 5$ sites, with $37.9 \%$.

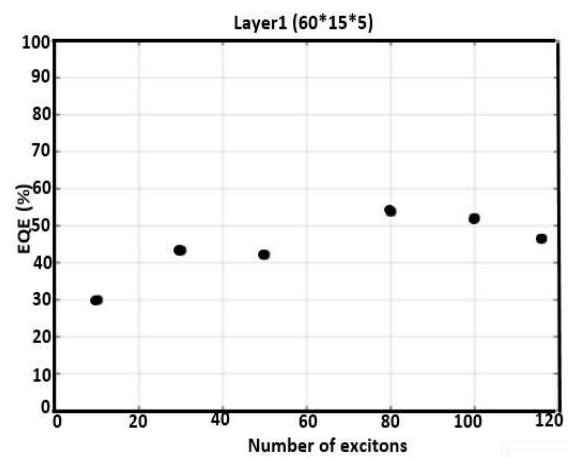

(a)

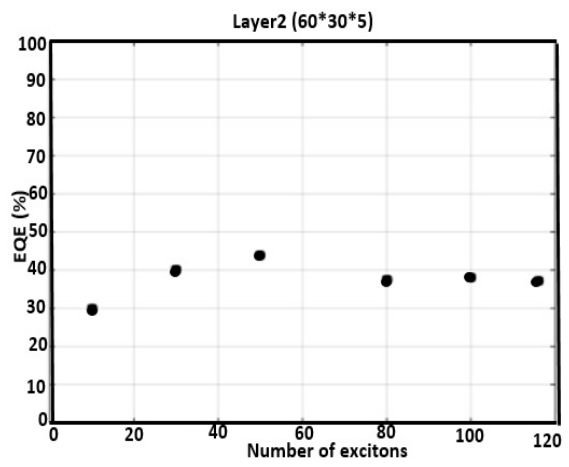

(b)

Fig. 9 External quantum efficiency according to the number of injected excitons. (a) $60 \times 15 \times 5$ site. (b) $60 \times 30 \times 5$ site

\section{Conclusion}

The results of numerical calculations of the transfer of electrons and holes in an organic checkered solar cell showed that the change in the structure dimensions of the layer affects the transfer of electrical charge carriers that play a significant role in the performance of the solar cell, it was concluded that the width of each layer and taking into account the effective parameters, the average number of electric carriers that have successfully reached their electrodes and help generate current in the external circuit has decreased due to the increased distance to the electrodes and the presence of a loss factor. Numerical calculations also showed that the $60 \times 15 \times 5$ sites, with a value of $42.63 \%$, has a higher external quantum efficiency than the $60 \times 30 \times 5$ sites, layer, with $37.9 \%$. It can be concluded that by increasing the layer width, the external quantum efficiency of the desired organic solar cell, due to increasing the distance of excitons to reach the separating boundary between the layers and reducing the number of electric charge carriers - which is directly related to external quantum efficiency - reduced.

\section{REFERENCES}

[1] K.K. Sadasivuni, K. Deshmukh, T. Ahipa, A. Muzaffar, M.B. Ahamed, S.K. Pasha, and M.A.-A. Al-Maadeed, "Flexible, biodegradable and recyclable solar cells: a review," J. Mater. Sci.: Mater. Electron, Vol. 30, pp. 951-974, 2019.

[2] A. Khalil, Z. Ahmed, F. Touati, and M. Masmoudi, "Review on organic solar cells," in: 2016 13th International Multi-Conference on Systems, Signals \& Devices (SSD), IEEE. Vol. 124, pp. 342-353, 2016.

[3] J. Ajayan, D. Nirmal, P. Mohankumar, M. Saravanan, M. Jagadesh, and L. Arivazhagan, "A review of photovoltaic performance of organic/inorganic solar cells for future renewable and sustainable energy technologies," Superlattices and Microstructures, Vol. 143, pp. 106549 (1-53), 2020.

[4] L. Yao, A. Rahmanudin, N. Guijarro, and K. Sivula, "Organic semiconductor based devices for solar water splitting," Adv. Energy Mater, Vol. 8, pp. 1802585 (1-18), 2018.

[5] M.P. Paranthaman, W. Wong-Ng, and R.N. Bhattacharya, Semiconductor materials for solar photovoltaic cells, Springer, 2016.

[6] W. Brütting, "Physics of Organic Semiconductors," Phys. Status Solidi A, Vol. 201, pp. 1031-1031, 2004.

[7] M. Wright and A. Uddin, "Organic - inorganic hybrid solar cells: A comparative review," Sol. Energy Mater. Sol. Cells, Vol. 107, pp. 87-111, 2012. 
[8] J. Asare, B. Agyei-Tuffour, O. Oyewole, V. Anye, D. Momodu, G. Zebaze-Kana, and W. Soboyejo, "Effects of Deformation on Failure Mechanisms and Optical Properties of Flexible Organic Solar Cell Structures," Trans Tech Publ. Vol. 1132, pp. 125-143, 2016.

[9] N. Sadoogi, A. Rostami, B. Faridpak, and M. Farrokhifar, "Performance analysis of organic solar cells: Opto-electrical modeling and simulation," Eng. Sci. Technol. an Int. J. Vol. 24, pp. 229-235, 2021.

[10] W. Farooq, A.D. Khan, A.D. Khan, and M. Noman, "Enhancing the power conversion efficiency of organic solar cells," Optik. Vol. 208, pp. 164093 (1-10), 2020.

[11]N. Tessler, and Y. Vaynzof, "Insights from Device Modeling of Perovskite Solar Cells," ACS Energy Lett. Vol. 5, pp. 1260-1270, 2020.

[12]X. Zhao, B. Mi, Z. Gao, and W. Huang, "Recent progress in the numerical modeling for organic thin film solar cells," Sci. China: Phys. Mech. Ast. Vol. 54, pp. 375-387, 2011.

[13]N. Xi, M. Zhang, and G. Li, Modeling and Control for Micro/Nano Devices and Systems, CRC Press. 2013.

[14]X. Xu, Monte Carlo simulation of charge transport in organic solar cells, Thesis, Duke University. 2012.

[15]U. Neupane, B. Bahrami, M. Biesecker, M.F. Baroughi, and Q. Qiao, "Kinetic Monte Carlo modeling on organic solar cells: Domain size, donor-acceptor ratio and thickness," Nano energy. Vol. 35, pp. 128-137, 2017.

[16]W. Tress, "Device Physics of Organic Solar Cells: Drift-Diffusion Simulation in Comparison with Experimental Data of Solar Cells Based on Small Molecules," Saechsische Landesbibliothek-Staats-und Universitaetsbibliothek Dresden. 2012.

[17]P.K. Watkins, A.B. Walker, and G.L. Verschoor, "Dynamical Monte Carlo modelling of organic solar cells: The dependence of internal quantum efficiency on morphology," Nano Lett. Vol. 5, pp. 18141818, 2005.

[18]A. Melianas and M. Kemerink, "Photogenerated Charge Transport in Organic Electronic Materials: Experiments Confirmed by Simulations," Advanced Materials. Vol. 31, pp. 1806004 (1-23), 2019.
[19] W. Ananda, "External quantum efficiency measurement of solar cell," International Symposium on Electrical and Computer Engineering, IEEE. Vol. 449, pp. 450-456, 2017.

[20] E.L. Lim, C.C. Yap, M.A.M. Teridi, C.H. Teh, and M.H.H. Jumali, "A review of recent plasmonic nanoparticles incorporated P3HT: PCBM organic thin film solar cells," Org. Electron, Vol. 36, pp. 12-28, 2016.

[21]F. Monestier, J.-J. Simon, P. Torchio, L. Escoubas, F. Flory, S. Bailly, R. de Bettignies, S. Guillerez, and C. Defranoux, "Modeling the short-circuit current density of polymer solar cells based on P3HT: PCBM blend," Sol. Energy Mater. Sol. Cells, Vol. 91, pp. 405-410, 2007.

[22] P.P. Boix, G. Garcia-Belmonte, U. Muñecas, M. Neophytou, C. Waldauf, and R. Pacios, "Determination of gap defect states in organic bulk heterojunction solar cells from capacitance measurements," Appl. Phys. Lett. Vol. 95, pp. 317- 321, 2009.

[23]F. Brioua, M. Remram, R. Nechache, and H. Bourouina, "Electrical and optical modeling of poly (3-hexylthiophene): [6, 6]-phenyl-C61 butyric acid methyl ester P3HT-PCBM bulk heterojunction solar cells," Appl. Phys. A, Vol. 123, pp.704 (1-10), 2017.

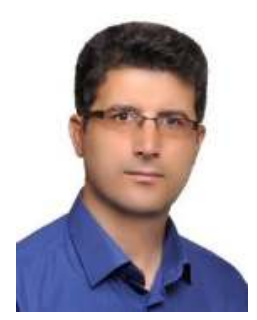

Mohsen Bahrami received the B.Sc degree in physics from Birjand university, Iran, M.Sc in atomic and molecular physics(laser) from Shahid Beheshti University, Iran, and PhD of Photonics from Laser and Plasma Research Institute, Shahid Beheshti University, Iran. He is currently an Assistance Professor at Lorestan University, Khorramabd, Iran. His main research interests are Biophotonics, lightmatter interaction and electrical charge and light transfer in different media. 


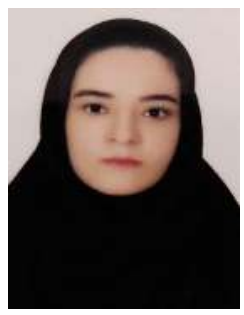

Nasrin Sepahvand received her B.Sc. degree in applied physics from Khorramabad Azad University, Iran, in 2007. She received her M.Sc degree in Atomic and molecular physics from Lorestan University, Iran, in 2018. Her research interests include photonic, optics and laser. 